meat-jack by its inherent meat-roasting power, and scorned the materialism of those wo sought to explain its action by some hidden mechanism in the chimney. Another possibility existed-namely, that Buchner had overlooked some constituent of blood serum, and that to this unknown constituent the bacteria-killing power of blood serum was due. It would lead me too far to attempt to detail the theoretical considerations that led me to suspect that a particular ferment-like proteid known as cell globulin $B$ was the substance in question. At any rate, $I$ tested its action on anthrax bacilli, and found that it possesses the power of destroying these microbes. I further found that similar substances were present not only in animals that are naturally immune against antbrax, but also in those that are susceptible to this disease. To those substances I have given the name of defensive proteids. In my published papers on this subject I have noted various similarities in the bactericidal action of these substances and that possessed by blood serum, and these resembiances are such as to leave little room for doubt that the bactericidal action of blood serum is due to the presence of these defensive proteids.

It is obvious that the mere presence of these bodies in the animal organism does not compel us to regard them as a means of resistance to microbe invasion. Before we can regard them as a real factor in the production of immunity it must be shown that the defensive proteid of a refractory animal is more active or is present in a larger quantity than is the case with an animal that is susceptible to a given disease. This very necessary proof I sought to obtain by a study of the defensive proteid of the rat. This animal is known to be highly resistant to anthrax. Behring, in 1888 , showed that its serum is more alkaline than that of any other animal that he examined; further, that it has the power of killing anthrax bacilli, which power is lost when the serum is neutralised. He came to the conclusion that the immunity of the rat to anthrax is due to this high alkalinity of its serum, but was unable to isolate the alkaline substance involved. Naturally, my work on defensive proteids enabled me to attack this question from a more favourable standpoint, and I soon found that this serum contained a proteid body possessing a well-marked alkaline reaction and a power of destroying anthrax bacilli. Further, when injected into mice, along with fully virulent anthrax spores, it would prevent the development of the disease. On the other hand, defensive proteids of animals susceptible to anthrax can exert no such protective power, and consequently these experiments indicate a difference in the mode of action of defensive proteids from immune and susceptible animals respectively. Further, the amount of defensive proteid present in a rat can be diminished by those causes which are known to be capable of lowering its power of resisting anthrax. For instance, Feser states that rats become susceptible to anthrax when fed on a vegetarian diet. I have obtained similar results with wild rats. The ordinary white rat, however, I have found to be generally refractory to anthrax on any diet, and always the defensive proteid can be obtained from its spleen and blood serum. With the wild rat this is not the case. In one experiment eight wild rats were used; of these, four were fed on bread and meat, the others on plain bread, for about six weeks. Then one rat of each lot was inoculated with anthrax; of these, the one that had been subjected to a bread diet succumbed. The remaining rats were killed, and it was found that while the spleens of the flesh-fed rats contained abundance of the defensive proteid, only traces of this substance could be obtained from the spleens of the rats that had been fed on bread alone. A similar result was obtained in other experiments.

These facts appear to me to prove that the defensive proteid of the rat deserves its name, in that it tends to preserve it from the attack of the anthrax microbe ; in other words, that this substance is, at any rate, a part cause of its immunity against anthrax.

Since the publication of my work on defensive proteids, Buchner has abandoned his view that the bacteria-killing power of blood serum is due to a remnant of vitality, and in a paper recently published he admits the importance of defensive proteids, and suggests for them the name "alexine." Certainly, if it were necessary to rechristen "defensive proteids," this name would be very appropriate. It would, however, be convenient to form names for the different classes of defensive proteids, and I do not think it would be premature to do so now. Defensive proteids appear to be ferment-like, albuminous bodies, and it is extremely unlikely that we shall for a considerable time be able to classify them by any other than physiological tests. From. this point of view it is possible to divide them into two classes : first, those occurring naturally in normal animals, and, secondly, those occurring in animals that have artificially been made immune. For these two classes I propose the names of "sozins"2 and "phylaxins." A "sozin" is a defensive proteid that occurs naturally in a normal animal. They have been found in all animals yet examined, and appear to act on numerous kinds of microbes or on their products. A "phylaxin" is a defensive proteid which is only found in an animal that has been artificially made immune against a disease, and which, so far as is yet known, only acts on one kind of microbe or on its products. Each of these classes of defensive proteids can obviously be further subdivided into those that act on the microbe itself and those that act on the poisons it generates. These subclasses I propose to denote by adding the prefixes "myco-"and "toxo-" to the class name. Thus, myco-sozins are defensive proteids occurring in the normal animal, which have the power of acting on various species of microbe. Toxo-sozins are defensive proteids also occurring in the normal animal, having the power of destroying the poisons produced by various microbes. Myco-phylaxins and toxo-phylaxins similarly will denote the two subclasses of the phylaxin group.

\section{THE HUMAN MOUTH AS A FOCUS OF INFECTION. ${ }^{1}$}

\section{By Dr. W. D. MILLER, BerliN.}

"DURING the last few years the conviction has growe continually stronger, among physicians as well as dentists, that the human mouth, as a gathering place and incubator of diverse pathogenic germs, performs a most significant rôle in the production of various disorders of the body, and that many diseases whose origin is enveloped in mystery, if they could be traced to their source, would be found to have originated in the oral cavity."

Dealing with the subject of diseases of the human body which have been traced to the action of mouth bacteria, he said: "In conformity with the nearly unanimous verdict of all recent investigations, decay of the teeth must be regarded as the most widespread of all parasitic diseases to which the human body is subject, and although, as far as the life of the patient is concerned, the prognosis is exceed. ingly good, and decay of the teeth may be pronounced one of the most trivial disturbances of the human economy, yet, if we take into consideration the results which follow a case of general decay, particularly in the mouths of young or weak persons, it often becomes a disease of a very grave nature. I venture to say that most dentists will agree with me that the havoc wrought by dental caries in the mouth of vast numbers of children, or even adults, among the lower classes is a much more serious thing than an attack of chicken-pox, rubeola, or even measles. Among the more immediate results of caries of the teeth may be mentioned diseases of the pulp and pericementum ; following these, alveolar abscess, which is produced by germs and their pro. ducts passing from the root canal through the foramen apicale into the surrounding tissue. Primarily of a local character, it is very frequently accompanied by general sym. ptoms of varying intensity, and sometimes attended by complications of most serious nature, death from alveolar abscess being by no means as rare an event as is usually supposed. Croupous pneumonia must be regarded as a disease in all probability closely dependent upon the condition of the mouth. The uniform results obtained by the investigators on the subject of pneumonia for the last fire years leave little room for doubt that the cause of this important disease is to be sought for in a species or group of microorganisms which are constantly present in the sputum of persons suffering from pneumonia, and very frequently even in the saliva of quite healthy persons. The infectious

2 I have to thank Dr. Donald MacAlister for his kind asolistance in helping me to coin these names.

1 Paper read in the Section of Bacteriology at the International Con gress of Hygiene. 
The Laxcer,] DR. MILler: The HUMAN MOU TH AS A FOCUS OF INFECtion. [August 15, 1891. 341

anginæ (tonsillitis, amygdalitis infectiosa, \&c.), with their severe complications, have been shown by the observations of Bouchard, Von Hoffmann, A. Fraenkel, Fiirbringer, Heubner, and Bahrat, Apolant, Leyden, \&c., to be due to the localisation of germs in the tonsils. Writers on the subject of angina Ludovici designate slight wounds or other breaks in the continuity of the mucous membrane, diseased teeth, the tonsils, and salivary ducts, \&c., as points of entrance for the specific germs of the disease, while the diseases of the maxillary sinus are known to owe their origin, in the great majority of cases, to diseased teeth. In connexion with pneumococcus abscesses, I call attention to the fact that the micrococci of sputum septicæmia, as shown by various authorities, possess invasive properties of the highest order, so that there is scarcely a part of the human body which may not fall a prey to their action. I may mention here parotitis, multiple subcutaneous abscesses, tonsillitis, otitis media, abscesses of the mastoid process, peritonitis, and meningitis." Many other infections were described in this connexion.

"The investigations of different bacteriologists during the last five years have brought to light a great number of pathogenic micro-organisms, some of which occur in the mouth with considerable frequency, others having been met with but a few times. On account of the large number of different micro-organisms commonly found in the human mouth, it is, with few exceptions, absolutely impossible to arrive at any conclusion regarding the presence or absence of any particular kind by a simple microscopic examination. Cultures on agar-agar also often fail of their purpose, since many pathogenic month bacteria do not grow on this culture medium, or they grow so slowly that they are soon overgrown and hidden by the more proliferous saprophytes of the mouth. Gelatine is still less adapted to the purpose. We must consequently have recourse to the animal body ffor the purpose of isolating such pathogenic micro-organisms as may be present in the saliva at the time of the examination. The person whose saliva was to be examined was always instructed to intermix the saliva by rubbing with the tip of the tongue against the cheeks and gums with dead epithelium and other films and deposits which are often clinging to the mucous membrane, and constantly carry enormous numbers of organisms. One or two of these drops were then injected into the abdominal cavity of a white mouse. Of the 111 mice thus operated upon, 27 died within 05 hours; 22 in 15 to 24 hours; 18 in 24 to 48 hours; 8 in 2 to 4 days; 9 in 4 to 8 days; 13 in 8 to 20 days; 4 in 20 to 40 days ; 10 being still healthy after the expiration of 30 days were put down as having escaped infection; though it is quite possible that one or the other of these 10, if kept longer under observation, would still have succumbed to the effects of the inoculation. In nearly all cases where the mice died within five days, the cause of death was found to be acute peritonitis or blood-poisoning, or both combined; whereas in the great majority of cases where death did not occur till after five days, no micro-organisms were found in the blood, death being due to local suppurative processes alone. We may accordingly make two grand subdivisions of the pathogenic mouth bacteria. The first includes those which produce speedy death through blood-poisoning, with comparatively slight local reaction; the second, those which induce fatal pyogenic processes at the point of injection. With very few exceptions, injections with the blood or peritoneal exudations of the deceased mice produced the same results as injections with saliva. In the 111 examinations above recorded, capsulated cocci or diplococci, which according to present usage would be called micrococci of sputum septicamia, were found in the blood of the mice 58 times, and, apart from these cases, three times in the peritoneal exudations-i.e, in all, sixty-one times. Micrococcus tetragenus was found in all twenty. six times. During the earlier experiments my attention was directed solely to the micrococcus of sputum septicemia, and I may have overlooked other organisms, so that in all probability the other species mentioned in reality oceur still oftener than indicated by my figures. Accordingly, the micrococcus of sputum septicæmia occurred sixty.one times; micrococcus tetragenus occurred twenty-eight times; megacoccus buccalis muciferens occurred four times; bacillus buccalis muciferens occurred three times; bacillus buccalis septicus occurred six times; bacillus pneumoniæ once. Besides these, various other micro-organisms of pathogenic significance were met with (apart from the pyogenic ones), which I was not able to study more closely. Twice streptococci were found in the blood.
" From the time that Pasteur discovered a deadly microorganism of the form of the figure 8 in the human saliva up to the present, developments regarding the nature and significance of the micrococci of sputum septicæmia have followed each other in rapid succession. Besides Pasteur, Raynaud and Lannelongue, Vulpian, Morgiggia and Marchiafava, Bochefontaine and Arthaud, Sternberg, Claxton, Gaglio and di Mattei, Griffin, Klein, A. Fraenkel, myself, and, more recently, scores of others have furnished contributions to the subject of the toxic properties of the saliva." Theauthor then proceeded to give in detail a description of the various micro organisms found in the human mouth capable of producing septicæmia. He also described the megaccoccus buccalis muciferens, bacillus buccalis muciferens, bacillus pneumoniæ (pneumobacillus), and the bacillus buccalis septicus. Continuing, he said : "Most observers agree that the micrococci of sputum septicæmia speedily lose their virulence when cultivated on artificial media. Ten days, seven, even five days are named as the maximum time for which the cocci remain virulent when cultivated on agar-agar, blood serum, \&c. The results which I have obtained do not quite accord with the above. A culture of the microcnccus of sputum septicemia Ir. from the blood of a mouse, on blood serum, dated May $6 \mathrm{th}$, which was kept for seven days at a temperature of $35^{\circ} \mathrm{C}$, subsequently at room-temperature, was used for innculating a mouse in the abdominal cavity on June 7th. The mouse died inside of twenty hours, showing a pure culture of the cocci in the blood. A culture of micrococcus II., twenty-nine days old, caused death in sixty-five hours; a culture forty days old failed to produce any effect. The cocci were found exceedingly resistive to the action of low temperature; a mouse, dead of an infection with micrococcus II., was hung up outside of the window for twenty-one days, between Dec. 22nd and Jan. 13th, the temperature ranging during nearly the whole time between $5^{\circ}$ and $15^{\circ} \mathrm{C}$. below zero. At the end of this time the mouse was thawed out, and an infection made with the blood resulted in the death of the infected animal inside of twenty-four hours. Regarding experiments relating to the question of immunity, it has been well established that immunity may be conferred upon animals by infecting them with material which has been so far weakened in its virulence that the onimals sicken, but recover. A subsequent infection, even with a fully virulent culture, may then be harmless. I have attempted to produce immunity (1) by injecting $0.5 \mathrm{ccm}$. of dog blood direct from the artery into the abdominal cavity of mice. Dogs being immune from sputum septicæmia, it was hoped thereby to confer immunity upon the mice. All experiments with dog blood, however, turned out negatively. The blood of a large American rabbit, which had been infected without showing any reaction, conferred a partial immunity upon mice, they dying not until the fifth or seventh day after infection, while the control mice died within twenty-four hours; (2) mice were fed for several days on large quantities of saccharine, with a view to so saturating them with this material that they would not furnish a suitable culture medium for the cocci ; results here also only negative; (3) a large number of antiseptic solutions were made use of, injecting the mice before or after, or both before and after the infection in the abdominal cavity, or subcutaneously with varying results, sometimes the death of the animal being hastened, sometimes slightly retarded. The only substance with which I attained positive results was a 1 per cent. solution of trichloride of iodine. If we inject a mouse subcutaneously with two drops of the water of condensation from a fresh agar-agar or blood serum culture, or with a slight quantity of diluted infectious blood, and follow up the injection through the same conule with $0.3 \mathrm{ccm}$. of $\mathrm{a}_{1} 1$ per cent. solution of trichloride of iodine (the maximal dose for a full.grown mouse), the animal will in most cases survive the infection, though it will lose a piece of skin as large as a finger-nail.

"The question to which I wish here to call particular attention concerns the measures which should be taken to prevent the undue growth of bacteria, pathogenic as well as non-pathogenic, in the mouth ; the ultimate object bein $g$ not alone to limit as far as possible the action of microorganisms and their products upon the teeth, but to keep within bounds as well the many various diseases which, we have seen, may result from a lack of proper care of the mouth. Not one of the many mouth washes with which the market is flooded makes even an approach towards accomplishing this end. For the purpose of disinfecting the mouth in cases of acute diseases, stomatitis, diphtheria, 
gangrene of the mouth, \&c., physicians usually have recourse to borax, boracic acid, chlorate of potash, perman. ganate of potash, lime water, salicylic acid, \&c., which, with the single exception of sallcylic acid, bave next to no action whatever upon the bacteria of the mouth, though some of them have undoubtedly an excellent cleansing action upon inflamed or suppurating surfaces, in virtue of which their use may be attended by very beneficial resulte." The author stated that sublimate, 1 in 2000 (eight tests), effected a marked diminution in the number of germs in one minute; the complete sterilisation, however, required on an average over five minutes. The efficacy of the sublimate was increased in a surprising degree by the addition of benzoic acid. Trichloride of iodine, 1 in 2000 (seven tests), required an average time of about a minute and a quarter, proving to be decidedly superior to the bichloride. It is also far less disagreeable than the latter-in fact, not at all disagreeable; it has, however, which must be considered as a great misfortune, an acid reaction, and is, therefore, not suited for daily use as a mouth wash. In the strength of 1 in 1500 (one test) the sterilisation was accomplished in forty seconds. After stating that in order to obtain practical results it was necessary to test the action of the solution on the bacteria in the month itself, rather than on pure cultures in bouillon, Dr. Miller described three methods of sterilisation, and enumerated the various agents he employed, with their respective values. He concluded that an examination of the results would soon convince one that there are very few substances at present in the dental materia medica which are available for disinfecting the human mouth. "A mouth wash which I recommended years ago, and which is decidedly superior to the best of the many su-called antiseptic mouth washes on the market, has the following construction :- $\mathrm{R}$ : Acid. benzoic, 3,0 ; tinct. encrlypt., 15, 0 ; alcohol. abs., 100, 0 ; ol. menth. pip., 0,75 ."

\section{THE PROPAGATION AND PREVENTION OF RABIES. ${ }^{1}$}

\section{'Yy GEORGE FLEMING, C B., LL.D., F.R.C.V.S.}

IT is now generally accepted by those who have most carefully studied the subject that rabies is a purely contagious disease, due to a virulent principle or specific agent, and is as a rule transmitted from a diseased to a healthy creature by inoculation. The specific agent, when introduced into the body of a susceptible animal, more particularly localises itself in the nervous system, and after a certain period, which is somewhat indefinite in accidental inoculation, but definite in experimental inoculation, causes such disturbance in the functions of the brain and spinal cord as to give rise to well-marked symptoms and a morbid condition that, it niay be asserted, invariably terminates in death. Inoculation and isfection occurthrough wounds which are generally inflicted by the teeth of creatures affected with rabies. Of all the domesticated animals which may become affected, the dog is certainly the one which is by far the most frequently attacked, and ic is the one which perpetuates the malady. It is undoubtedly the chief, if not the only, propagator of the scourge in this and other countries; and it is therefore exceedingly probable-nay, certain-that if the canine species were completely freed from rabies the disease would be no longer knuwn. Recognising this fact, and in view of the constant virulency of the saliva of rabid animals, and consequently of danger from their bites, and the serious consequences resulting therefrom, the importance of sanitary measures applicable to this animal cannot be over-estimated. In the interests of mankind, no less than in those of the domesticated animals, and particularly of the dog itself, this very alarming, most painful, and always fatal disorder should be suppressed. And of all the known contagious diseases, rabies is the one which can be most easily and quickly extinguished, provided proper measures are prescribed, and are carried stringently and energetically into operation.

The measures necessary for the extinction of the disease must be based on the fact that the dog is the chief-nay,

1 Paper read in the Section of the Relation of Diseases of Animals to those of Man at the International Congress of Hygiene. sole-propagator of rabies; that the contagium or infecting material is conveyed from the diseased to the healthy by means of bites (other means are so exceedingly rare that they may be left out of consideration); and that to free a country from it, all rabid and suspected dogs should be destroyed, certain restrictions maintained for a limitec period, and only dogs from other countries exempt from rabies allowed admission. Dogs coming from infected or suspected countries should undergo a period of quarantine equal at least to the longest period of latency of the disease. That the disease can be limited or altogether suppressed by the enforcement of proper measures there is an abund. ance of evidence to prove. Sweden, Norway, Switzerland, Baden, Prussia, Bavaria, Wurtemburg, and other countries have been freed from it by such measures; while countries in which it has never been seen, as Australia and New Zealand, are preserved from its invasion by such precautionary measures as quarantine of imported dogs, or, as in Sweden, total prohibition of importation of dogs.

That the dog is the chief sufferer from, and, it may be asserted, the sole propagator, of the malady is amply proved by every-day experience; but the following table, sbowing the number of cases of rabies in England during the four years 1887-90, and the number and species of animals affected, affords further evidence :--

\begin{tabular}{|c|c|c|c|c|c|c|}
\hline \multicolumn{3}{|c|}{ Find of animal. } & $\frac{188 \%}{217}$ & $\begin{array}{r}1888 . \\
160\end{array}$ & $\begin{array}{r}1889 . \\
-12\end{array}$ & 1890. \\
\hline $\begin{array}{l}\text { Dogs .. } \\
\text { Cattle } \\
\text { Sheep.. } \\
\text { Swine.. } \\
\text { Horses } \\
\text { Deer .. }\end{array}$ & $\begin{array}{l}. . \\
\ddot{.} \\
\ddot{.} \\
\ddot{.}\end{array}$ & 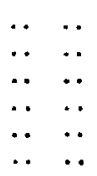 & $\begin{array}{r}217 \\
11 \\
5 \\
3 \\
4 \\
257\end{array}$ & $\begin{array}{r}160 \\
2 \\
7 \\
- \\
5 \\
2\end{array}$ & $\begin{array}{r}312 \\
9 \\
11 \\
4 \\
4 \\
-\end{array}$ & $\begin{array}{r}129 \\
2 \\
- \\
7 \\
2 \\
-\end{array}$ \\
\hline & al & .. $\quad$. & 497 & 176 & 840 & 184 \\
\hline
\end{tabular}

It will be observed that in 1888 and 1889,90 and 91 per cent. of the animals reported as rabid were dogs, and in 1890 the percentage was more than 96 . The animals other than dogs were infected by rabid dogs, and these infected each other; as the mad dog has always the strongest tendency to attack its own species, it follows that this furnishes by far the largest proportion of victims. The suppressive sanitary police measures are, in the order of their importance-(1) Destruction of all dogs which are rabid, or whish are suspected of being or becoming rabid; (2) the seizure and, if need be, destruction of all ownerless and wandering dogs ; (3) all other dogs to wear a properly construeted and well-fitting muzzle while rabies prevails, and also for a period equal to the longest interval of latency after the malady has been suppressed; (4) the imposition of a tax upon all dogs.

1. The necessity for the destruction of all rabid dogs is so self-evident that it need not be further insisted upon. The disease is incurable, causes much suffering, and the affected $\mathrm{dog}$ is, so long as it lives, so great a danger to other creatures that its speedy destruction is imperative. A suspected dog may be defined to be one that presents symptoms resembling those of rabies, or which bas been in conditions that have rendered infection probable or possible.

2. Wandering dogs are the chief agents in the propagation of rabies, for the reason that they are moreexposed to contamination than those which are properly cared for, and also because rabid dogs, even when carefully guarded, seek to wander from home. In proportion as wandering dogs abound in infected countries, so is the disease prevalent. This is a well-estaio lished fact. Therefore, in order to limit the propagation of rabies, all wandering dogs should be secured, and, if not. claimed within a certain period, destroyed.

3. Rabies being transmitted, as a rule, by the bites of dogs, and as in countries in which the disease prevails there can be no certainty when a dog is not infected, if it at all times be prevented from inflicting wounds by its teeth, it is evident that the danger of extension o the malady must be reduced at least to a minimum. Dogs can be hindered from biting by causing them to wear a properly con. structed muzzle; and if it fits well, it should cause very little, if any, inconvenience to them. The value of the muzzle in suppressing rabies has been demonstrated on many occasions, and in serious outbreaks of the disease its introduction has always coincided with a diminution in the number of cases and the eventual extinction of the scourge. Its use is also coincident with a decrease 